\title{
The adult sex ratio of European regions predicts female, but not male, subjective well-being
}

\author{
Thomas Richardson ${ }^{1}$ (D) \\ Accepted: 10 March 2021 / Published online: 27 March 2021 \\ (C) The Author(s) 2021
}

\begin{abstract}
In recent years, researc in subjective well-being has found several ecological factors that may underpin societal differences in happiness. The adult sex ratio, the number of males relative to females in an environment, influences many behaviours in both humans and non-human animals. However, the possible influence of the sex ratio on subjective well-being has received little attention. I investigated the relationship between the adult sex ratio and subjective well-being in over 29,000 respondents from 133 regions of Europe. I find that women report lower subjective well-being in areas with more female-biased sex ratios, but males' well-being was unaffected. I did not find that the sex ratio influences the sex specific probability of marriage, or marriage rates overall. I also find that increased population density is associated with lower well-being. Drawing from sociological and evolutionary theories, I suggest that results may be due to females' decreased bargaining power in the dating market.
\end{abstract}

Keywords Operational sex ratio $\cdot$ Adult sex ratio $\cdot$ Subjective well-being $\cdot$ Population density

\section{Introduction}

The factors underlying subjective well-being (SWB) are a topic of immense study within the behavioural sciences. SWB is influenced by individual-level factors, but also societal and ecological ones. Individual-level factors that predict SWB include income, extroversion, and positive affect (reviewed in Diener et al., 2018), as well as status and success, (Curhan et al., 2014; Wirtz \& Scollon, 2012) and self-esteem (Brown, 2010). The relationship between individual level factors and SWB may differ in magnitude with culture (reviewed in Suh \& Choi, 2018). Factors that predict societal differences in SWB include living in an 'open', individualistic society (Krys et al., 2019) with effective democracy (Orviska et al., 2014), where gains in economic wealth are relatively evenly distributed (Oishi \& Kesebir, 2015), such as through progressive taxation (Oishi et al., 2012). SWB can be influenced by the interaction between cultural and individual factors, with individuals being happier when their personalities or emotions 'fit' with those in the culture around them (Fulmer et al., 2010; De Leersnyder et al., 2014; further examples are given in

Thomas Richardson

thomas.richardson@manchester.ac.uk

1 The University of Manchester, Oxford Rd, Manchester M13 9PL, UK
Oishi \& Gilbert, 2016). Ecological predictors of low life satisfaction include high population density ( $\mathrm{Li} \&$ Kanazawa, 2016), and a demanding climate (Fischer \& Van de Vliert, 2011). Here I investigate the effects of an ecological variable fundamentally related to the behaviour of most animals including humans: the adult sex ratio.

The adult sex ratio, or numbers of adult males for every adult female in a population (Schacht et al., 2017) is a primary determinant of the availability of potential partners in an environment (Emlen \& Oring, 1977 ). When there are few potential partners in a local environment, competition to secure a partner can be fierce. Finding and keeping mates are universal human motives (Ko et al., 2020) and failure in this area can be a source of despair. While this is to some degree self-evident, it is also supported by a large literature showing that being married predicts higher SWB (Haring-Hidore et al., 1985; Diener, 2000; but see Stutzer \& Frey, 2006). The sex ratio has been shown to impact a wide variety of behaviours (reviewed in Maner \& Ackerman, 2020), from promiscuous behaviour (Kandrik et al., 2015; Millar et al., 2019; Schacht \& Borgerhoff Mulder, 2015) to facial hair fashion (Barber, 2001) to female economic empowerment (Teso, 2019; Grosjean \& Khattar, 2019) to economic activity (Chang \& Zhang, 2015; Griskevicius et al., 2012; Wei \& Zhang, 2011).

Despite vast literatures on both topics, few studies have investigated the link between the adult sex ratio and SWB. Those that have, primarily focus on depression and suicide. 
Kuroki (2014) found that the sex ratio of Japanese prefectures influenced male, but not female suicide rates. There were more male suicides in prefectures where there were fewer women. The effect was especially strong in older men. Interestingly this effect was not mediated by participant's marital status suggesting it was not entirely explained by a lack of marriage prospects. Moore et al. (2020) also found that the sex ratio was positively related to the male suicide rate in a longitudinal analysis of a single Scottish region, though only in times when the unemployment rate was high. When unemployment was low, the suicide rate was negatively related to the male sex ratio. Zhou et al. (2013) found that depression rates for Chinese men and women were higher in more malebiased regions, though the effect was stronger among males. However, the study sampled only 12 regions, none of which showed female-biased sex ratios. Moreover, they did not account for the fact that regions' sex ratios are not independent of each other, an issue known as Galton's problem (Pollet et al., 2014). Lastly, Grosjean and Brooks (2017) found that some areas of Australia in the 1700s had strongly male-biased populations due to an influx of convicts from the British empire, which they showed had a lasting impact on the culture of these regions. Couples living in regions that were more malebiased several hundred years ago were happier today as a result. Taken together, there seems to be no clear consensus on how the sex ratio affects sex specific SWB.

It is also unclear what mechanism would drive sex ratio related changes in subjective well-being. One mechanism is that sex ratio imbalances increase the SWB of the rarer sex, as they have greater 'bargaining power' in the dating market. This gives them more options for partnership and more power within current partnerships (Du et al., 2015; Guttentag \& Secord, 1983). Similarly, the more abundant sex has reduced power in the dating market, and this may decrease SWB. A particularly explicit example of this was when a large influx of men into Taiwan in the 1950's led to a sharp increase in bride price relative to dowry, suggesting that men decreased in value as marriage partners and women increased in value (Francis, 2011). In polygynous cultures, men also have more wives when women are abundant (Pollet \& Nettle, 2009). Both men and women expect that men should pay more for romantic gifts to females in cities with male-biased sex ratios compared to female-biased ones (Griskevicius et al., 2012). There is mixed evidence as to whether women secure better partners in more male-biased sex ratios (Harknett, 2008; Lichter et al., 1995; Pollet \& Nettle, 2008). This 'bargaining power mechanism' predicts that male SWB should be negatively related to the sex ratio and female SWB positively related to the sex ratio.

A second mechanism by which the sex ratio could influence subjective well-being is through marriage rates and family stability. In slightly male-biased areas, marriage rates can increase for both sexes (Schacht \& Kramer, 2016), there are fewer divorces (Abramitzky et al., 2011; Uggla \& Andersson, 2018) and as such family instability is lower (Copping \& Campbell, 2015). Marriage in turn increases SWB for both the men and women involved. This 'marriage mechanism' is related to the bargaining power mechanism, in that marriage rates are positively related to the sex ratio because females can use their increased bargaining power to elicit greater commitment from men (Schacht \& Borgerhoff Mulder, 2015). However, these 2 mechanisms diverge in their predictions for male SWB; the bargaining power mechanism predicts male SWB would decline in male-biased sex ratios, whereas the marriage mechanism predicts it would increase. This is partially supported by Grosjean and Brooks (2017) though their effect was driven by ancestral, rather than modern sex ratios. If both a bargaining power and a marriage mechanism are at work they may cancel out, having no net effect on male SWB. We can test the marriage mechanism by observing if the effect of the sex ratio on SWB disappears when marital status is controlled for.

The third mechanism is the indirect effect of sex ratio imbalances on SWB through effects on the local societal variables, such as through crime rates, societal attitudes, or the economy. The sex ratio is correlated with many such variables (e.g. life expectancy, see Jin et al., 2010), though causality often isn't clear (Kandrik et al., 2015). There is a large literature investigating the role of the ASR on levels of violence and homicide, and while the literature is mixed, overall the pattern seems to be that a surplus of males decreases violence (Schacht et al., 2014). One reason for this may be increased marital stability and decreased numbers of female headed households in male-biased areas, a known risk factor for violent crime (a hypothesis tested in Messner \& Sampson, 1991). Male-biased sex ratios may also decrease sex crimes (Schacht et al., 2016) though this may not be true in all cultures (Bose et al., 2013). Grosjean and Brooks (2017) argued that areas of Australia that were historically more male-biased developed cultures of stronger gender roles, with females experiencing more discrimination and participating less in the workforce, while enjoying more leisure time (an argument developed further in Grosjean \& Khattar, 2019). They argue that this culture persists, and explains modern Australian couples' greater SWB and life satisfaction.

Additionally, the sex ratio may influence SWB through its effects on economic variables. Evidence from China and Taiwan indicate that in male-biased sex ratios, men are more likely to become entrepreneurs in the hope that economic success will make them more attractive to women, leading to sharp increases in local GDP and saving (Chang \& Zhang, 2015; Wei \& Zhang, 2011). Increases in GDP may lead to increased SWB (Easterlin, 1974; Lin et al., 2014; Veenhoven, 1991). These effects will all be reversed in a female-biased sex ratio, though this has yet to be demonstrated as previous research has been conducted in Asian countries which have consistently (and often grossly) malebiased sex ratios. On the other hand, Americans of both sexes 
living in cities with relatively male-biased sex ratios have more credit cards on average and have larger debts (Griskevicius et al., 2012), which may reduce SWB by increasing financial stress. If economic prosperity is positively related to the sex ratio, we might see downstream positive effects of the sex ratio on SWB for both sexes. Crucially, we can distinguish this 'economic mechanism' from the above bargaining power and marriage mechanisms as this predicts increased SWB for both sexes regardless of whether they are married, but that the effect would be mediated by measures of local economic conditions.

The present study investigates the effect of sex ratio on SWB using data from waves 6 and 7 of the European Social Survey (European Social Survey (ESS ERIC), 2013; 2015). Using GDP, unemployment, and marriage rate data, I explore the possible mechanisms by which the sex ratio might influence SWB. There is a general lack of studies of female-biased sex ratios in the literature (discussed in Schacht \& Kramer, 2016), as malebiased sex ratios are far more common in well-documented datasets. There is a need to understand the consequences of female biased sex ratios, as the sex ratios of many regions around the world are currently trending towards female bias (Dyson, 2012). To address this issue, I employ data from European regions within countries, which I find show significant female-biased ASRs. I also attempt to replicate previous studies that show a negative effect of population density on SWB. In both cases I fit multilevel models to person-level data to allow for inferences about individuals (avoiding the ecological fallacy) and to minimise Galton's problem (see Pollet et al., 2014; and 2017 for a discussion of these problems).

\section{Methods}

\section{Sample}

The present study combined data from the 2012 and 2014 European Social Surveys (ESS6 and ESS7 respectively). I included data on respondents from the 133 regions across 11 countries for whom data on region-level gross domestic product (GDP), unemployment rate and numbers of males and females were available for both years. Data was only analysed from respondents between the ages of 16 and 65 . Regions are defined using the Nomenclature of Territorial Units for Statistics (NUTS, abbreviated from the original French) framework by Eurostat, the statistical office of the European Union. The final sample was 29681 respondents: $15381 \mathrm{fe}-$ males and 14300 males.

\section{Measures}

To create a holistic measure of subjective well-being, I selected all items present in both ESS6 and ESS7 that were directly relevant to happiness and well-being. This resulted in 6 questions, measuring how satisfied respondents were with their life overall (10 point scale), as well as how often in the last week they enjoyed life, felt depressed, happy, lonely, or sad (all 4 point scales). These showed moderate intercorrelations, with the average correlation among scores on any 2 questions being $r=|0.42|$. Variables were rescaled to all be between 1 and 10, and averaged to create a composite SWB score that also ran between 1 and 10 for ease of interpretation. Cronbach's alpha showed the scale had good internal reliability (alpha $=0.75)$.

To measure the local adult sex ratio, I used data compiled by Eurostat on the percentage of the adults $>16$ years old that were male in each region. This is preferable to a ratio of males to females as it is symmetrical and more intuitive to interpret. However, I will refer to this "percentage male" as adult sex ratio (ASR) throughout in line with previous studies (Ancona et al., 2017; Kramer et al., 2017). I included gross domestic product as measured per 1 million Euros at market prices (GDP) and percentage of the total population unemployed as proxies for the state of the local economy.

To investigate the effects of the adult sex ratio on marital status I collapsed the 5-option marital status question into 2 options. "legally married" and "in a legally registered civil union" were coded as "married". "legally separated", "legally divorced", "widowed" and "none of the above" were coded as "unmarried". In many countries (such as the UK until 2018) civil unions are only available to same-sex couples. For this reason, it may be preferable to remove them from the dataset as their behaviour is unlikely to influenced by the sex ratio to the extent to which they may not be heterosexual. However, they were included in the data, as removing them would unbalance the dataset because same sex respondents not in a civil partnership would remain in the dataset.

\section{Analysis and Results}

All data and code required to reproduce the analyses are available on the open science framework https://osf.io/yjpn3/.

The distribution of the ASR for each wave is given below, where an even sex ratio (50\% male) is marked in each case. A paired t-test showed that the mean ASR slightly, but significantly decreased between $2012($ mean $=49.09 \%)$ and 2014 (mean $=48.99 \%), \mathrm{t}(115)=4.73, p<0.0001$, Cohen's $d=$ -0.07 . One sample t-tests showed that in both 2012 and 2014, the sex ratio significantly deviated from $50 \%$ male, with female biased sex ratios on average. For 2012: $\mathrm{t}(122)=$ $-14.24, p<0.0001, d=1.45$. For 2014: $t(125)=-17.05$, $p<0.0001 d=1.55$ (Fig. 1).

Unless otherwise stated, in all analyses that follow, data from both waves (2012 or 2014) was combined to maximise statistical power. The data was clustered in several ways; individuals were clustered within regions, which themselves 
Fig. 1 Distributions of regional sex ratios, for ESS6 and ESS7. The orange line indicates an even sex split of $50 \%$ male
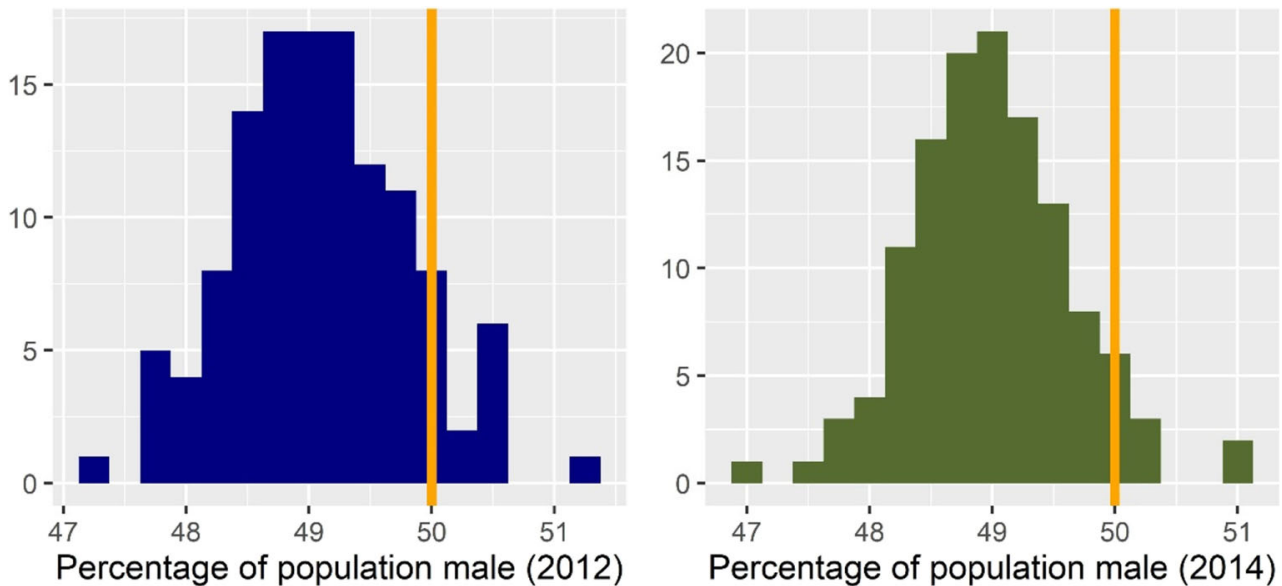

were clustered in countries, which were clustered in survey waves. To account for temporal clustering, survey wave was included in all analyses as a fixed effect control variable (coded as $2012=0$ or $2014=1$ ). To account for spatial clustering, mixed effects models were used, with random intercepts of region and country. Some analyses were aggregated at the region level, and as such only have a random intercept of country. Random slopes were included for predictors of interest to reduce risk of false positives (Barr et al., 2013). Generally, region-level variables of interest were given slopes on country, as were interactions between individual-level variables and region level variables. When a mixed effect model failed to converge, the bobyqa optimiser was used, which always resulted in convergence. All confidence intervals reported are $95 \%$ confidence intervals.

First, I fitted a linear mixed-effects model on data aggregated to the regional level (1 data point per region, per year) predicting sex ratio from GDP, the unemployment rate and population density. GDP and population density were skewed so were natural log transformed. Survey wave was added as a control variable, and a random intercept of country was included. The unemployment rate $(b=0.03, C I=0.01-0.05, p=$ $0.002)$, and $\ln$ (GDP) $(b=-0.17, C I=-0.24--0.11, p<$ $0.0001)$ but not $\ln$ (population density) $(p=0.41)$ significantly predicted the ASR. Areas with lower unemployment and higher GDP showed more female-biased sex ratios.

Next, I investigated the effect of population density on subjective well-being. I fitted a linear mixed-effects model with random intercepts of region and country. $\mathrm{Ln}$ (Population density), sex and their interaction were entered as predictors as well as $\ln$ (GDP), unemployment rate and survey wave as control variables. I fitted a random slope of the sex * density interaction on country. $\operatorname{Ln}(\mathrm{GDP})$ was not significant ( $p=$ $0.55)$ so was removed and the model refitted. The interaction between sex and population density was also non-significant $(p=0.30)$, so it was removed along with it's random slope, and the random slope of population density on country was added. In this final model, there was a significant negative effect of population density on subjective well-being ( $b=$ $-0.03, C I=-0.06--0.006, p=0.01)$.

I then attempted to replicate effects that show increased marriage rates for both sexes under male-biased sex ratios. Marriage data was not available for 871 individuals, so they were excluded. I fitted a logistic mixed-effects model predicting married status from ASR controlling for respondent sex and survey wave. A random slope of ASR on country was included. There was no effect of ASR on the probability of being married overall $(p=0.80)$, but women were more likely to be married than men in the sample $(\mathrm{OR}=1.10, C I=1.05$ $1.16, p<0.0001)$. As such an interaction effect between participant sex and ASR was added, with a slope of this variable on country replacing the slope of ASR on country. However, in this new model there was no significant interaction between sex and ASR $(p=0.27)$. Overall there was no effect of the ASR on marriage probability for males or females.

I then fitted linear mixed-effects models to test whether SWB was influenced by ASR, and whether factors such as economic conditions and marital status mediated any relationships. As different hypotheses predicted the presence or absence of sex differences in effects, models were fitted separately for each sex. Results are given in Table 1. The first model predicted SWB from regional ASR, with survey wave as a control variable and a random slope of ASR on country. For males, ASR showed no significant effect on $\operatorname{SWB}(p=$ 0.12 ), but in females a higher (male-biased) ASR was significantly associated with greater SWB $(p=0.02)$. Increasing the sex ratio by 1 percentage point increased female wellbeing by an average of 0.14 on a 10 point scale.

I tested whether the interaction between ASR and marital status predicted subjective well-being for each sex separately. In this model, age and survey wave were added as control variables and the random slope of ASR on country was replaced with random slopes of the ASR - marital status interaction on country. There was no significant interaction between ASR and marital status for males $(p=0.78)$ or females $(p=0.64)$. Next, I tested whether the effect of ASR on female 
Table 1 The effects of the \% of males in a region (ASR), marital status and their interactions on SWB for men and women. Models also control for survey wave (not displayed)

\begin{tabular}{lllll}
\hline & & \multicolumn{2}{l}{ Wellbeing score } \\
\cline { 3 - 5 } & & $b$ & $95 \% C I$ & $p$ \\
\hline Males & & & & \\
Model 1 & ASR (\% male) & 0.06 & $-0.01-0.19$ & 0.1 \\
Mode12 & ASR (\% male) & 0.04 & $-0.02-0.11$ & 0.21 \\
& Marital status & 0.49 & $0.44-0.54$ & $<\mathbf{0 . 0 0 0 1}$ \\
& Age & -0.01 & $-0.02--0.012$ & $<\mathbf{0 . 0 0 0 1}$ \\
& ASR * Marital status & -0.01 & $-0.08-0.11$ & 0.77 \\
Females & & & & \\
Model 1 & ASR (\% male) & 0.14 & $0.05-0.24$ & $\mathbf{0 . 0 1}$ \\
Model 2 & ASR (\% male) & 0.11 & $0.03-0.18$ & $\mathbf{0 . 0 0 3}$ \\
& Marital status & 0.44 & $0.39-0.50$ & $<\mathbf{0 . 0 0 0 1}$ \\
& Age & -0.01 & $-0.02--0.012$ & $<\mathbf{0 . 0 0 0 1}$ \\
& ASR * Marital status & 0.02 & $-0.06-0.10$ & 0.61 \\
Model 3 & ASR (\% male) & 0.12 & $0.04-0.21$ & $\mathbf{0 . 0 0 2}$ \\
& Marital status & 0.44 & $0.39-0.49$ & $<\mathbf{0 . 0 0 0 1}$ \\
& Age & -0.01 & $-0.02--0.012$ & $<\mathbf{0 . 0 0 0 1}$ \\
\hline
\end{tabular}

SWB was mediated by a main effect of marital status. This was not necessary for males as there was no main effect of ASR. This was a model predicting SWB from ASR, marital status, with age and survey wave as control variables. The effect of ASR on female SWB in the final model remained significant when marital status was controlled for $(p=0.002)$. In this model each $1 \%$ increase in the ASR increased female wellbeing by an average of 0.12 points on a 10 point scale. It was unnecessary to test whether the unemployment rate or GDP mediated the relationship between ASR and SWB, because a high ASR was associated with higher female SWB but higher unemployment and lower GDP, meaning that they cannot explain the effect of ASR.

Finally, I included fitted models including ASR and ASR ${ }^{2}$, to test the exploratory hypothesis that sex ratio deviations in either direction would have the same effects on subjective well-being. In both cases, a random slope of $\mathrm{ASR}^{2}$ on country was included. This effect was non-significant in both males $(p=0.51)$ or females $(p=0.22)$.

\section{Robustness Checks}

Having found a significant effect of sex ratio on female, but not male well-being, I decided to subject both effects to robustness checks. For each check I report whether results changed qualitatively or not (whether $p$ values $<0.05$ changed to $>0.05$ and vice versa) and how much the coefficient of the ASR effect changed. The effect of population density was also subject to robustness effects but is reported in supplementary material 1 as it is not the main effect of interest. To summarise: the effect of population density passed all robustness checks.

As an alternative composite of SWB, I ran a principal components analysis with varimax rotation on the 6 SWB variables and extracted the first component, which captured 52\% of the variance. I re-ran analyses using this as the dependent variable. Results were all qualitatively the same.

The percentage of males in a region tends to be lower in areas with aging populations, as men die at greater rates than women at every life stage (Hollingshaus et al., 2019). This means analyses may be confounded by the age distributions of regions. Including the median age of each region would be the best way to address, but this was not available. Instead I calculated the median age of the respondents from each region as a proxy of region-wide median age. The ESS samples representatively with respect to age for each given country, though age distributions of the respondents within regions may not be perfectly representative of the age distribution of their region. Nonetheless, regional median age correlated negatively with regional ASR $r(247)=-0.23, p=0.0002$, suggesting that it may be a reasonable proxy. This 'regional median age' variable was entered as a control variable into the mixed effects models above predicting SWB from $\%$ male for males and females. The results did not change qualitatively, with the coefficient for the effect of the ASR on female subjective wellbeing remaining the same $(b=0.14)$ and the coefficient for males increasing from $b=0.06$ to $b=0.08$ but remaining non significant $p=0.12$ ).

The sample contains different numbers of respondents for each region, varying from below 30 for some regions to over 850 for other regions. While mixed effects models account for this imbalance through shrinkage, it is possible that the results may be biased by particularly large regions (who contribute the most datapoints), or small regions (which may have extreme values if their data is not representative due to sampling error). As such I re-ran the female model 1 (the primary finding of the analysis) including the number of datapoints for a given region as a covariate. The effect was unchanged $(b=$ $0.15, p=0.01$ ), I also re-ran the model excluding a) the smallest 10 regions and $b$ ) the largest 10 regions, the latter of which representing 11,983 participants. In both cases the effect of sex ratio remained relatively unchanged $(b=0.13$, $p=0.03$ and $b=0.17, p=0.01$ respectively).

\section{Discussion}

I examined the influence of the adult sex ratio across regions of Europe on partnership and subjective well-being (SWB) using data from rounds 6 and 7 of the European Social Survey. Overall, most areas of Europe showed significantly female-biased sex ratios. Areas that had relatively more women had lower unemployment and higher GDP. The probability 
of being married was uninfluenced by the sex ratio. Men's SWB was unaffected by the sex ratio, while women showed increased SWB in areas of Europe where there were more men. I also found that higher population density was associated with lower SWB.

The current findings that the adult sex ratio (ASR) is associated with SWB is consistent with previous work (Kuroki, 2014; Moore et al., 2020; Zhou et al., 2013; but see Grosjean \& Brooks, 2017). However, I find that female, but not male SWB is influenced by the ASR. This contrasts to previous work that primarily showed effects of the sex ratio on male, but not female SWB (Kuroki, 2014). There were relatively few male-biased sex ratios in the European regions analysed here. By contrast, previous work in China showed very few femalebiased areas (e.g. Zhou et al., 2013). European men and Chinese women have more romantic options than their opposite sex peers but this does not seem to increase their wellbeing. It may be that only some individuals show increased happiness, while others show decreased happiness, which then average out. For example, European men may be better able to realise their desires for casual sex (Kandrik et al., 2015), but the increased mating success is unlikely to be evenly distributed, so the benefits may not be realised by all. Some of these men may also suffer increased violence, which has been documented in female-biased sex ratios (Schacht et al., 2014).

One possible way to reconcile these hypotheses is if a lack of mates makes one unhappy, but an abundance of mates does not make one happier. Favourable sex ratios allows us to select a better mate than we otherwise would have, but unfavourable sex ratios risk us not finding a partner at all. It is well established that poverty decreases wellbeing, while being rich contributes much less to happiness, and a similar relationship may exist for availability of romantic partners. Alternatively, differences in results may also be due to fact that previous studies investigated suicide, which usually indicates an extreme lack of SWB but is not the same construct. This emphasises the need for studies of the same topic in diverse adult sex ratios of both male and female bias.

The mechanism behind the increased SWB of women in male-biased areas is unclear. The null effect for the $\mathrm{ASR}^{2}$ term suggests that it is not simply that any sex ratio deviations form 50:50 affect well-being. The data do not suggest a 'marriage mechanism', as the effect remained significant when marital status was controlled for (though the $p$ value did decrease almost to non significance from 0.02 to 0.04 ), and ASR did not interact with marital status. As these areas have higher unemployment and lower GDP, it also cannot be due to economic factors, because this would predict a lower SWB, not a higher one as seen here. It is possible that women are happier due to changes in an unobserved societal variable correlated with the sex ratio (i.e. the 'cultural mechanism'), such as violent crime rates (Schacht et al., 2014). Alternatively, it could suggest a 'bargaining power mechanism' more general to intersexual relations, where women enjoy advantages in a male-biased sex ratio regardless of marital status. Unmarried women may also have increased bargaining power in male biased sex ratios. For example, in Grosjean and Khattar (2019) women living in ancestrally male-biased areas of Australia enjoyed more leisure time (though attained fewer positions of authority at work). One limitation of the current work is that it is correlational; it is possible that reverse causation is driving the results. It may be that happier women preferentially move to male-biased areas, or conversely that less happy women move to female biased areas. It is hard to see why this would be the case, but not impossible, so a definitive mechanism for these results is currently lacking.

The variation in the percent of the population that is male (i.e. the sex ratio) across European regions was small, with a standard deviation of 0.71 percentage points. Likely this is partially the reason the effect sizes found were small. Population dynamics may also work to dampen the effect: if the sex ratio is so imbalanced it makes one sex very unhappy, members of that sex may migrate to areas with better prospects. This will smooth out serious sex ratio imbalances. Bearing this in mind, it is perhaps remarkable that the relatively small deviations from an equal sex ratio senn here have any effect at all. In understanding the size of the effect found, comparing the relative size of the model coefficients could be illuminating: an increase in the percentage male of $4 \%$ (roughly the difference between the highest and lowest ASR in the sample) would produce an equivalent increase in female SWB as being married, or a 11-year decrease in age, equivalent to 0.44 points on a 10 point well-being scale. Additionally, we can compare the effect size to other higher-level variables: a $1 \mathrm{SD}$ increase in regional ASR on female SWB ( 0.1 points on a 10 point scale) is almost double the effect of a 1SD increase in country level income inequality $(0.056$ points on a 10 point scale, taken from Oishi \& Kesebir, 2015 study 1, scaled to a 10 point scale from original 4 point scale). However, the 1SD effect of the ASR is far smaller than the effect of living in an open society, which can be as high as 0.29 points on a 10 point scale (calculated from Krys et al., 2019). These comparisons suggests that the effects are neither groundbreaking nor trivial. In many countries such as China, far more extreme sex ratios are observed, with areas being 55\% male not uncommon (Dyson, 2012). In these countries, the sex ratio may exert a substantial impact on SWB, though caution must be taken generalising beyond the range of the data and to different cultures.

I did not replicate the finding commonly seen in the literature that marriage rates increase for both sexes in male-biased areas (Abramitzky et al., 2011; Schacht \& Kramer, 2016). Furthermore, I did not find that the probability of being married diverged for men and women in uneven adult sex ratios. Assuming the percentage of people who want to marry is the same across regions, changes in the sex ratio must result in diverging probabilities of marriage for the sexes. This is because the number of people that have no marriage partner available to them increases. However, if the number of people 
who want to marry changes systematically with the sex ratio, the probability of each sex being married can change in the same direction. For example, if women desire marriage more than men do, and in male-biased sex ratios men must increasingly conform to female preferences to secure a mate, the proportion of men married may increase overall. This would happen because the increase in 'excess unmarriageable males' is smaller than the number of males who feel pressured to marry because of the male-biased sex ratio but may not have married otherwise. I see neither pattern in this data, perhaps due to low statistical power from relatively low variation in sex ratios across European regions. There may also be factors that the present analysis did not account for.

In both samples the adult sex ratio was related to local economic conditions; more female-biased areas were associated with lower unemployment, and higher GDP. It is unclear whether the link between ASR and economic conditions is causal, and if so, which direction the causality goes. Previous studies from China show opposite effects; a malebiased ASR is related to increased economic prosperity and larger GDP increases over time (Chang \& Zhang, 2015; Wei $\&$ Zhang, 2011). This was not seen in the current study. The sex ratios in Europe and China diverge in opposite ways; most European regions studied were female-biased, whereas China has an alarmingly high male bias. Quite different cultural pressures create these differences, with China's sex ratio being driven by a combination of a culture of son-preference and the availability of sex-selective abortion (Hesketh \& Xing, 2006), neither of which have been documented in Europe. There is good reason to predict that a male-biased sex ratio should affect economic variables, as men value wealth more than women do (Schwartz \& Rubel, 2005) and there seems to be a universal pattern across cultures of men competing for mates by accumulating resources (Buss, 1988). Additionally, some evidence suggests they are more likely to do this when they perceive sex ratios to be unfavourable (Griskevicius et al., 2012). How local economies may be influenced by the sex ratio is an interesting and potentially important topic for future research.

This research has implications for female subjective wellbeing in areas that have female biased sex ratios. For example, Similarly, sex ratios may become strongly female biased after war (Brainerd, 2017) or in communities with high male crime and incarceration (Pouget, 2017). University campuses across many countries have female biased sex ratios, and women from the these colleges are appropriately pessimistic about their dating prospects (Uecker \& Regnerus, 2010). This may contribute college age women's lower subjective well-being (Leino \& Kisch, 2005), though we leave this possibility to further research.

I found that people reported lower SWB in areas with higher population density. This held despite controlling for economic conditions. This is in line with previous work by
Li and Kanazawa (2016), who argued that humans evolved in lower population densities than are found in modern environments (Dunbar, 1993) and this 'evolutionary mismatch' creates unhappiness. This hypothesis is difficult to test, as it technically refers to the number of people an individual must interact with on a regular basis, of which population density is only a proxy. Moreover, the effect of population density is hard to separate from the effects of living in an urban environment, which may influence happiness in many ways independent of population density. Indeed, Li and Kanazawa (2016) argue that population density is the mediator behind the finding that people who live in rural areas are often happier than urbanites (Easterlin et al., 2011). Neither the current study nor Li and Kanazawa (2016) have the data to separate these variables. Nonetheless it is an intriguing hypothesis that may warrant further investigation, particularly with the growth and increasing urbanisation of the world population.

One limitation of the current study is that regions varied in their size and so may vary in how good they are as a proxy of the mating market an individual faces. The 'correct' level of analysis for examining mating markets is unclear; very large units of analyses (e.g. entire countries with millions of people) may smooth out meaningful variation in sex ratios, whereas highly granular units (e.g. the neighbourhood level) may produce large amounts of noise if participants can seek mates outside the unit of analysis. For this reason, I encourage other scholars to investigate the relationship between sex ratio and SWB at a variety of levels, such as counties within countries, or at the city level, to confirm the present findings.

In conclusion, I show that the female-biased sex ratios seen across regions of modern Europe are associated with decreased female SWB but have no association with male SWB. The increased SWB of women in male-biased areas is unexplained by marriage rates or economic conditions. The mechanisms by which the sex ratio influences SWB are currently an open question.

Supplementary Information The online version contains supplementary material available at https://doi.org/10.1007/s12144-021-01619-5.

Acknowledgements I would like to thank Rob Brooks, Tucker Gilman and the Gilman Lab for comments on a draft of this manuscript. I also thank the Engineering and Physical Science Research Council of the UK (EPSRC) for funding this research.

Declarations All data was collected in accordance with The Declaration on Professional Ethics of the International Statistical Institute and all respondents gave informed consent.

Conflict of Interest The author declares no conflict of interest.

Open Access This article is licensed under a Creative Commons Attribution 4.0 International License, which permits use, sharing, 
adaptation, distribution and reproduction in any medium or format, as long as you give appropriate credit to the original author(s) and the source, provide a link to the Creative Commons licence, and indicate if changes were made. The images or other third party material in this article are included in the article's Creative Commons licence, unless indicated otherwise in a credit line to the material. If material is not included in the article's Creative Commons licence and your intended use is not permitted by statutory regulation or exceeds the permitted use, you will need to obtain permission directly from the copyright holder. To view a copy of this licence, visit http://creativecommons.org/licenses/by/4.0/.

\section{References}

Abramitzky, R., Delavande, A., \& Vasconcelos, L. (2011). Marrying up: The role of sex ratio in assortative matching. American Economic Journal: Applied Economics, 3(3), 124-157.

Ancona, S., Dénes, F. V., Krüger, O., Székely, T., \& Beissinger, S. R. (2017). Estimating adult sex ratios in nature. Philosophical Transactions of the Royal Society B: Biological Sciences, 372(1729), 20160313.

Barber, N. (2001). Mustache fashion covaries with a good marriage market for women. Journal of Nonverbal Behavior, 25(4), 261-272.

Barr, D. J., Levy, R., Scheepers, C., \& Tily, H. J. (2013). Random effects structure for confirmatory hypothesis testing: Keep it maximal. Journal of Memory and Language, 68(3), 255-278.

Bose, S., Trent, K., \& South, S, J. (2013). The effect of a male surplus on intimate partner violence in India. Economic and Political Weekly, $48(35)$.

Brainerd, E. (2017). The lasting effect of sex ratio imbalance on marriage and family: Evidence from world war II in Russia. Review of Economics and Statistics, 99(2), 229-242.

Brown, J. D. (2010). Across the (not so) great divide: Cultural similarities in self-evaluative processes. Social and Personality Psychology Compass, 4, 318-330.

Buss, D. M. (1988). The evolution of human intrasexual competition: Tactics of mate attraction. Journal of Personality and Social Psychology, 54(4), 616-628.

Chang, S., \& Zhang, X. (2015). Mating competition and entrepreneurship. Journal of Economic Behavior and Organization, 116, 292 309.

Copping, L. T., \& Campbell, A. (2015). The environment and life history strategies: Neighborhood and individual-level models. Evolution and Human Behavior, 36(3), 182-190.

Curhan, K. B., Levine, C. S., Markus, H. R., Kitayama, S., Park, J., Karasawa, M., Kawakami, N., Love, G. D., Coe, C. L., Miyamoto, Y., \& Ryff, C. D. (2014). Subjective and objective hierarchies and their relations to psychological well-being: A US/Japan comparison. Social Psychological and Personality Science, 5(8), 855-864.

De Leersnyder, J., Mesquita, B., Kim, H., Eom, K., \& Choi, H. (2014). Emotional fit with culture: A predictor of individual differences in relational well-being. Emotion, 14(2), 241-245.

Diener, E. (2000). Subjective well-being: The science of happiness and a proposal for a national index. American Psychologist, 55(1), 34-43.

Diener, E., Lucas, R, E., \& Oishi, S. (2018). Advances and open questions in the science of subjective well-being. Collabra. Psychology, $4(1): 15$.

Du, J., Wang, Y., \& Zhang, Y. (2015). Sex imbalance, marital matching and intra-household bargaining: Evidence from China. China Economic Review, 35, 197-218. https://doi.org/10.1016/j.chieco. 2014.11.002

Dunbar, R. I. (1993). Coevolution of neocortical size, group size and language in humans. Behavioral and Brain Sciences, 16(4), 681694
Dyson, T. (2012). Causes and consequences of skewed sex ratios. Annual Review of Sociology, 38, 443-461.

Easterlin, R. A. (1974). Does economic growth improve the human lot? Some empirical evidence. In Nations and households in economic growth (pp. 89-125). Elsevier.

Easterlin, R. A., Angelescu, L., \& Zweig, J. S. (2011). The impact of modern economic growth on urban-rural differences in subjective well-being. World Development, 39(12), 2187-2198.

Emlen, S. T., \& Oring, L. W. (1977). Ecology, sexual selection, and the evolution of mating systems. Science, 197(4300), 215-223.

European Social Survey ERIC (ESS ERIC). (2013). European Social Survey (ESS), Round 6-2012. https://doi.org/10.21338/NSDESS6-2012

European Social Survey ERIC (ESS ERIC). (2015). European Social Survey (ESS), Round 7-2014. https://doi.org/10.21338/NSDESS7-2014

Fischer, R., \& Van de Vliert, E. (2011). Does climate undermine subjective well-being? A 58-nation study. Personality and Social Psychology Bulletin, 37(8), 1031-1041.

Francis, A. M. (2011). Sex ratios and the red dragon: Using the Chinese communist revolution to explore the effect of the sex ratio on women and children in Taiwan. Journal of Population Economics, 24(3), 813-837.

Fulmer, C. A., Gelfand, M. J., Kruglanski, A. W., Kim-Prieto, C., Diener, E., Pierro, A., \& Higgins, E. T. (2010). On "feeling right" in cultural contexts: How person-culture match affects self-esteem and subjective well-being. Psychological Science, 21(11), 1563-1569.

Griskevicius, V., Tybur, J. M., Ackerman, J. M., Delton, A. W., Robertson, T. E., \& White, A. E. (2012). The financial consequences of too many men: Sex ratio effects on saving, borrowing, and spending. Journal of Personality and Social Psychology, 102(1), 69-80

Grosjean, P., \& Brooks, R. C. (2017). Persistent effect of sex ratios on relationship quality and life satisfaction. Philosophical Transactions of the Royal Society B: Biological Sciences, 372(1729), 20160315. https://doi.org/10.1098/rstb.2016.0315.

Grosjean, P., \& Khattar, R. (2019). It's raining men! Hallelujah? The long-run consequences of male-biased sex ratios. The Review of Economic Studies, 86(2), 723-754. https://doi.org/10.1093/restud/ rdy025.

Guttentag, M., \& Secord, P. F. (1983). Too many women?: The sex ratio question. Sage Publications, Inc.

Haring-Hidore, M., Stock, W. A., Okun, M. A., \& Witter, R. A. (1985). Marital status and subjective well-being: A research synthesis. Journal of Marriage and the Family, 47, 947-953.

Harknett, K. (2008). Mate availability and unmarried parent relationships. Demography, 45(3), 555-571.

Hesketh, T., \& Xing, Z. W. (2006). Abnormal sex ratios in human populations: Causes and consequences. Proceedings of the National Academy of Sciences, 103(36), 13271-13275.

Hollingshaus, M., Utz, R., Schacht, R., \& Smith, K. R. (2019). Sex ratios and life tables: Historical demography of the age at which women outnumber men in seven countries, 1850-2016. Historical Methods: A Journal of Quantitative and Interdisciplinary History, 52(4), 244 253.

Jin, L., Elwert, F., Freese, J., \& Christakis, N. A. (2010). Preliminary evidence regarding the hypothesis that the sex ratio at sexual maturity may affect longevity in men. Demography, 47(3), 579-586.

Kandrik, M., Jones, B. C., \& DeBruine, L. M. (2015). Scarcity of female mates predicts regional variation in men's and women's sociosexual orientation across US states. Evolution and Human Behavior, 36(3), 206-210.

Ko, A., Pick, C. M., Kwon, J. Y., Barlev, M., Krems, J. A., Varnum, M. E., Neel, R., Peysha, M., Boonyasiriwat, W., \& Brandstätter, E. (2020). Family matters: Rethinking the psychology of human social motivation. Perspectives on Psychological Science, 15(1), 173-201. 
Kramer, K. L., Schacht, R., \& Bell, A. (2017). Adult sex ratios and partner scarcity among hunter-gatherers: Implications for dispersal patterns and the evolution of human sociality. Philosophical Transactions of the Royal Society B: Biological Sciences, 372(1729), 20160316.

Krys, K., Uchida, Y., Oishi, S., \& Diener, E. (2019). Open society fosters satisfaction: Explanation to why individualism associates with country level measures of satisfaction. The Journal of Positive Psychology, 14(6), 768-778.

Kuroki, M. (2014). The effect of sex ratios on suicide. Health Economics, 23(12), 1502-1510.

Leino, E. V., \& Kisch, J. (2005). Correlates and predictors of depression in college students: Results from the spring 2000 national college health assessment. Journal of Health Education, 36(2), 66-74.

Li, N. P., \& Kanazawa, S. (2016). Country roads, take me home... to my friends: How intelligence, population density, and friendship affect modern happiness. British Journal of Psychology, 107(4), 675-697.

Lichter, D. T., Anderson, R. N., \& Hayward, M. D. (1995). Marriage markets and marital choice. Journal of Family Issues, 16(4), 412431.

Lin, C.-H. A., Lahiri, S., \& Hsu, C.-P. (2014). Happiness and regional segmentation: Does space matter? Journal of Happiness Studies, 15(1), 57-83.

Maner, J, K., \& Ackerman, J, M. (2020). Ecological sex ratios and human mating. Trends in Cognitive Sciences, 24(2), 98-100.

Messner, S. F., \& Sampson, R. J. (1991). The sex ratio, family disruption, and rates of violent crime: The paradox of demographic structure. Social Forces, 69(3), 693-713.

Millar, M. G., Westfall, R. S., \& Walsh, M. (2019). The moderating effects of mate-value on the relationship between perceived sex ratio and mating strategies. Personality and Individual Differences, 145, $39-43$.

Moore, F, R., Macleod, M., Starkey, C., Krams, I., \& Roy, T. (2020). Effects of the sex ratio and socioeconomic deprivation on male mortality. Archives of Suicide Research, 24(3), 435-449.

Oishi, S., \& Gilbert, E. A. (2016). Current and future directions in culture and happiness research. Current Opinion in Psychology, 8, 54-58.

Oishi, S., \& Kesebir, S. (2015). Income inequality explains why economic growth does not always translate to an increase in happiness. Psychological Science, 26(10), 1630-1638.

Oishi, S., Schimmack, U., \& Diener, E. (2012). Progressive taxation and the subjective well-being of nations. Psychological Science, 23(1), 86-92.

Orviska, M., Caplanova, A., \& Hudson, J. (2014). The impact of democracy on well-being. Social Indicators Research, 115(1), 493-508.

Pollet, T. V., \& Nettle, D. (2008). Driving a hard bargain: Sex ratio and male marriage success in a historical US population. Biology Letters, 4(1), 31-33.

Pollet, T. V., \& Nettle, D. (2009). Market forces affect patterns of polygyny in Uganda. Proceedings of the National Academy of Sciences, 106(7), 2114-2117.

Pollet, T. V., Tybur, J. M., Frankenhuis, W. E., \& Rickard, I. J. (2014). What can cross-cultural correlations teach us about human nature? Human Nature, 25(3), 410-429. https://doi.org/10.1007/s12110014-9206-3.

Pollet, T. V., Stoevenbelt, A. H., \& Kuppens, T. (2017). The potential pitfalls of studying adult sex ratios at aggregate levels in humans.
Philosophical Transactions of the Royal Society B: Biological Sciences, 372(1729), 20160317.

Pouget, E. R. (2017). Social determinants of adult sex ratios and racial/ ethnic disparities in transmission of HIV and other sexually transmitted infections in the USA. Philosophical Transactions of the Royal Society B: Biological Sciences, 372(1729), 20160323.

Schacht, R., \& Borgerhoff Mulder, M. (2015). Sex ratio effects on reproductive strategies in humans. Royal Society Open Science, 2(1), 140402.

Schacht, R., \& Kramer, K. L. (2016). Patterns of family formation in response to sex ratio variation. PLoS One, 11(8), e0160320.

Schacht, R., Rauch, K. L., \& Mulder, M. B. (2014). Too many men: The violence problem? Trends in Ecology and Evolution, 29(4), 214 222.

Schacht, R., Tharp, D., \& Smith, K. R. (2016). Marriage markets and male mating effort: Violence and crime are elevated where men are rare. Human Nature, 27(4), 489-500.

Schacht, R., Kramer, K. L., Székely, T., \& Kappeler, P. M. (2017). Adult sex ratios and reproductive strategies: A critical re-examination of sex differences in human and animal societies. Philosophical Transactions of the Royal Society B: Biological Sciences, 372(1729), 20160309. https://doi.org/10.1098/rstb.2016.0309.

Schwartz, S. H., \& Rubel, T. (2005). Sex differences in value priorities: Cross-cultural and multimethod studies. Journal of Personality and Social Psychology, 89(6), 1010-1028.

Stutzer, A., \& Frey, B. S. (2006). Does marriage make people happy, or do happy people get married? The Journal of Socio-Economics, 35(2), 326-347.

Suh, E. M., \& Choi, S. (2018). Predictors of subjective well-being across cultures. Handbook of well-being. DEF Publishers.

Teso, E. (2019). The long-term effect of demographic shocks on the evolution of gender roles: Evidence from the transatlantic slave trade. Journal of the European Economic Association, 17(2), 497534.

Uecker, J. E., \& Regnerus, M. D. (2010). Bare market: Campus sex ratios, romantic relationships, and sexual behavior. The Sociological Quarterly, 51(3), 408-435.

Uggla, C., \& Andersson, G. (2018). Higher divorce risk when mates are plentiful? Evidence from Denmark. Biology Letters, 14(9), 20180475.

Veenhoven, R. (1991). Is happiness relative? Social Indicators Research, 24(1), 1-34.

Wei, S.-J., \& Zhang, X. (2011). Sex ratios, entrepreneurship, and economic growth in the People's Republic of China (no. 0898-2937). National Bureau of Economic Research.

Wirtz, D., \& Scollon, C. N. (2012). Culture, visual perspective, and the effect of material success on perceived life quality. Journal of CrossCultural Psychology, 43(3), 367-372.

Zhou, X. D., Li, L., Yan, Z., \& Hesketh, T. (2013). High sex ratio as a correlate of depression in Chinese men. Journal of Affective Disorders, 144(1-2), 79-86.

Publisher's Note Springer Nature remains neutral with regard to jurisdictional claims in published maps and institutional affiliations. 